\title{
Hsa_circ_0033155: A potential novel biomarker for non-small cell lung cancer
}

\author{
XIAOHUA GU ${ }^{1}$, GANG WANG ${ }^{2}$, HUI SHEN ${ }^{1}$ and XIAOYUN FEI ${ }^{1}$ \\ ${ }^{1}$ Department of Respiratory Medicine, Shanghai Jiaotong University Affiliated Sixth People's Hospital, \\ Shanghai 200233; ${ }^{2}$ State Key Laboratory of Bioreactor Engineering, School of Biotechnology, \\ East China University of Science and Technology, Shanghai 200237, P.R. China
}

Received February 8, 2018; Accepted June 29, 2018

DOI: $10.3892 /$ etm.2018.6565

\begin{abstract}
Circular RNAs (circRNAs) are a novel type of endogenous RNAs and increasing evidence have uncovered the important role of circRNA in tumor progression; however, the function of circRNAs in non-small cell lung cancer (NSCLC) remains largely unclear. In the present study, it was demonstrated that the expression level of hsa_circ_0033155 was significantly downregulated in NSCLC tissue and the expression of hsa_circ_0033155 correlated with lymphatic metastasis. In order to further investigate the possible role of hsa_circ_0033155 in NSCLC progression, circRNA was overexpressed in NSCLC cells and it was observed that the overexpression of hsa_circ_0033155 significantly decreased cell proliferation, colony formation and migration, and elevated the level of phosphatase and tensin homolog deleted on chromosome 10, a tumor suppressor in many types of tumor. In conclusion, hsa_circ_0033155 may serve as a prospective biomarker for detection and a promising therapeutic target for NSCLC.
\end{abstract}

\section{Introduction}

Lung cancer is the leading cause of cancer-associated mortality worldwide and $>80 \%$ cases are non-small cell lung cancer (NSCLC) (1). Despite consistent advances in the diagnosis and molecular targeted therapy techniques for effective control of lung cancer, the clinical outcomes remain unsatisfactory (2). The number of mortalities due to lung cancer is estimated to be 154,050 in 2018, accounting for $25.27 \%$ of all cancer-associated mortalities (1), and the overall 5-year survival rate of patients with lung cancer remains poor at $<20 \%$ (3). These poor statistics are primarily due to the fact that the majority of patients

Correspondence to: Dr Xiaoyun Fei, Department of Respiratory Medicine, Shanghai Jiaotong University Affiliated Sixth People's Hospital, 600 Yishan Road, Shanghai 200233, P.R. China

E-mail: fxyfeifei@sina.com

Key words: circular RNA, biomarker,hsa_circ_0033155,phosphatase and tensin homolog deleted on chromosome 10, non-small cell lung cancer present with advanced disease stage at the time of diagnosis, at which point, the optimal time to perform surgery has passed (4). Therefore, improving the rate of early diagnosis is particularly important in increasing the opportunities for surgical intervention of patients with NSCLC, and identification of promising diagnostic markers for early detection is urgent.

Circular RNA (circRNA), which widely exists in mammalian cells, has received increasing focus in endogenous noncoding RNA research (5). One important characteristic of circRNA is the high level of stability that provides great possibilities as a biological marker $(5,6)$. Additionally, emerging studies have suggested the aberrant expression of circRNAs in various diseases, including esophageal squamous cell carcinoma, gastric cancer and pancreatic ductal adenocarcinoma $(7,8)$. It has been suggested that circRNAs act as microRNA (miRNA) sponges, regulating gene expression, and serving important roles in tumorigenesis and tumor progression $(7,8)$. The aforementioned evidence indicates the potential value of circRNAs as novel biomarkers and therapeutic targets for cancer diagnosis and treatment. At present, the roles of circRNAs in the progression of NSCLC remain unclear $(9,10)$. The majority of valuable circRNAs, including hsa_circ_0000190 in gastric cancer (11), are potential biomarkers with an increased sensitivity and specificity compared with classic biomarkers for the diagnosis of malignancies and includes carcinoembryonic antigen and CA19-9. circRNAs have not been comprehensively investigated in the field of heterogeneous NSCLC (12).

In the present study the circRNA, hsa_circ_0033155 was investigated, which was demonstrated to be downregulated in NSCLC tissues according to our previous circRNA microarray analysis (unpublished data). To the best of our knowledge, the present study revealed for the first time that the expression level of hsa_circ_0033155 was significantly downregulated in NSCLC tissues. Subsequently, the association between hsa_circ_0033155 expression and clinicopathological parameters of NSCLC was analyzed. In order to study the role of hsa_circ_0033155 in NSCLC progression, the biological functions of hsa_circ_0033155 were investigated. In addition, the levels of phosphatase and tensin homolog deleted on chromosome 10 (PTEN), a modulator of cell survival, which has been identified as a tumor suppressor in various tumor types (13), were evaluated. 


\section{Materials and methods}

Specimens. All clinical samples were collected from the respiratory department of Shanghai Jiaotong University Affiliated Sixth People's Hospital (Shanghai, China) between April 2013 and June 2016. NSCLC tissues and their matched adjacent non-tumorous tissues $5 \mathrm{~cm}$ from the edge of the tumor were obtained from 40 surgical patients, (males, 23; females, 17; age range, 36-57 years). All tissue specimens were immediately preserved in RNA-fixer reagent (Bioteke Corporation, Beijing, China) following resection and stored at $-80^{\circ} \mathrm{C}$ prior to use in subsequent experiments. No patients received radiotherapy, chemotherapy or targeted therapy prior to surgery. Tumor size was calculated using the widest diameter determined on computerized tomography (CT) images. Magnetic resonance imaging (MRI) and CT findings were used to diagnose lymphatic metastasis and to evaluate tumor differentiation, respectively. Tumor histological grading and staging were performed according to the World Health Organization classification criteria and the Tumor Node Metastasis system of the International Union Against Cancer (7th edition). The present study was approved by the Human Research Ethics Committee of Shanghai Jiaotong University and written informed consent was obtained from all patients.

Cell culture. NSCLC cell lines HCC827 and H1975 were purchased from American Type Culture Collection (Manassas, VA, USA) and normal human bronchus epithelium cell line BEAS-2B and NSCLC cell lines PC9 and H1650 were from Shanghai Suer Biological Technology Co., Ltd. (Shanghai, China). The cell lines were cultured in RPMI-1640 supplemented with $10 \%$ fetal bovine serum (FBS) and $1 \%$ penicillin/streptomycin (all Invitrogen; Thermo Fisher Scientific, Inc., Waltham, MA, USA). All cells were incubated at $37^{\circ} \mathrm{C}$ with $5 \% \mathrm{CO}_{2}$.

Transient transfection. PC9 and H1650 were cultured in the above mentioned medium, seeded in 6-well plates at a density of $4.0 \times 10^{5}$ cells/well and incubated at $37^{\circ} \mathrm{C}$ in $5 \% \mathrm{CO}_{2}$. The following day, cells were transfected with the overexpression vector (14 $\mu \mathrm{g})$ for hsa_circ_0033155 (pLCDH-ciR-hsa_circ_0033155; Geneseed Biotech Co., Ltd., Guangzhou, China) using Lipofectamine 2000 (Invitrogen; Thermo Fisher Scientific, Inc.) following the manufacturer's protocol. Negative control (NC) groups were established by transfection with the control vector (pLCDH-ciR; Geneseed Biotech Co., Ltd.). Cells were cultured for $24 \mathrm{~h}$ following transfection. For selection of the successfully transfected clones, cells were seeded in 6-well plates (4,000 cells/well) and treated with $400 \mu \mathrm{g} / \mathrm{ml} \mathrm{G} 418$ (Sigma-Aldrich; Merck KGaA, Darmstadt, Germany) at $37^{\circ} \mathrm{C}$ for 10 days. Medium containing G418 was replaced every 3 days. Positive clones were verified using reverse transcription-quantitative polymerase chain reaction (RT-qPCR).

Total RNA extraction and RT- $q P C R$. Total RNA from paired NSCLC and adjacent non-tumorous tissues as well as cell lines were extracted using TRIzol reagent (Invitrogen; Thermo Fisher Scientific, Inc.) according to the manufacturer's protocol. Then, ReverTra Ace (Toyobo Life Science, Osaka, Japan) was applied to reverse transcribe total RNA into single-stranded cDNA following the manufacturer's protocol. qPCR was performed using the SYBR Premix Ex Taq $^{\text {TM }}$ II kit (Takara Biotechnology Co., Ltd., Dalian, China) in a final volume of $10 \mu \mathrm{l}$ containing $0.5 \mu \mathrm{l} \mathrm{cDNA}, 0.5 \mu \mathrm{l}$ each primer and $5 \mu \mathrm{l}$ SYBR Green. Primers were synthesized by Sangon Biotech Co., Ltd., (Shanghai, China) and their sequences were as follows: GAPDH, forward 5'-GGAGCGAGATCCCTC CAAAAT-3' and reverse 5'-GGCTGTTGTCATACTTCT CATGG-3'; hsa_circ_0033155, forward 5'-GGGGTCAGG AAAGAAACTGC-3' and reverse 5'-TGTCGTCTTCTTGCA TCTGG-3'; PTEN, forward 5'-TGGATTCGACTTAGACTT GACCT-3' and reverse 5'-GGTGGGTTATGGTCTTCAAAA GG-3'. Thermal cycling was as follows: $94^{\circ} \mathrm{C}$ for $5 \mathrm{~min}$, then 42 cycles at $94^{\circ} \mathrm{C}$ for $5 \mathrm{sec}$ and $60^{\circ} \mathrm{C}$ for $1 \mathrm{~min}$. Data were analyzed using the $2^{-\Delta \Delta \mathrm{Cq}}$ method (14), whereby a higher $\Delta \mathrm{Cq}$ values indicates a lower expression of hsa_circ_0033155.

Cellproliferationassay.CellsinNCandhsa_circ_0033155groups were seeded in 96-well plates at a density of $3.5 \times 10^{3}$ cells/well and incubated for 1-7 days, under the culture conditions stated above. A total of $5 \mathrm{mg} / \mathrm{ml}$ MTT (Sigma-Aldrich; Merck KGaA) was subsequently added to the wells and cultured at $37^{\circ} \mathrm{C}$ for $3 \mathrm{~h}$, then the supernatant was discarded and dimethyl sulfoxide (Sigma-Aldrich; Merck KGaA) was added. Subsequently, the absorbance was detected at $492 \mathrm{~nm}$ to determine the proliferation rate. Cell proliferation was evaluated using the following equation: Proliferation rate $=\mathrm{A}($ sample $) / \mathrm{A}$ (control); where $\mathrm{A}$ indicates the absorption measured at $492 \mathrm{~nm}$ and the control describes the sample analyzed at day 0 .

Colony formation assay. Cells in NC and hsa_circ_0033155 groups were seeded in 6 -well plates $\left(6.0 \times 10^{2}\right.$ cells/well) and incubated at $37^{\circ} \mathrm{C}$ for 14 days. The medium was replaced every 2 days during the culture. Subsequently, the plates were washed with PBS and the colonies were fixed with $100 \%$ methanol at room temperature (RT) for $15 \mathrm{~min}$, then stained with $1 \%$ crystal violet at RT for $30 \mathrm{~min}$. Finally, stained colonies with $>50$ cells were counted under a light microscope at x200 magnification.

Migration assay. The migration assay was performed in cells from NC and hsa_circ_0033155 groups using 24-well Transwell inserts (8- $\mu \mathrm{m}$ pore size; EMD Millipore, Billerica, MA, USA) according to the manufacturer's protocol. A total of $1.0 \times 10^{4}$ cells were suspended in serum-free RPMI-1640 and seeded in the upper chamber, whereas RPMI-1640 containing $10 \%$ FBS was added to the lower chamber. Following incubation at $37^{\circ} \mathrm{C}$ for $24 \mathrm{~h}$, cells in the upper surface were removed and cells remaining on the bottom surface were fixed with $100 \%$ methanol at RT for $15 \mathrm{~min}$, followed by staining with $1 \%$ crystal violet at RT for $30 \mathrm{~min}$. Finally, stained cells were photographed under a light microscope at x200 magnification and counted in at least five randomly selected fields.

Western blot analysis. NC and hsa_circ_0033155 cell lysates were prepared using cell lysis buffer (Sigma-Aldrich; Merck $\mathrm{KGaA})$ and then centrifuged $\left(13,800 \mathrm{x} \mathrm{g} ; 5 \mathrm{~min} ; 4^{\circ} \mathrm{C}\right)$. Total protein concentration was detected using Bradford protein assay kit from Hangzhou MultiSciences (Lianke) Biotech Co., Ltd. (Hangzhou, China) following the manufacturer's protocol. Protein $(20 \mu \mathrm{g})$ was separated on $12 \%$ SDS-PAGE gels and subsequently transferred to polyvinylidene fluoride membranes. The membranes were incubated with the 

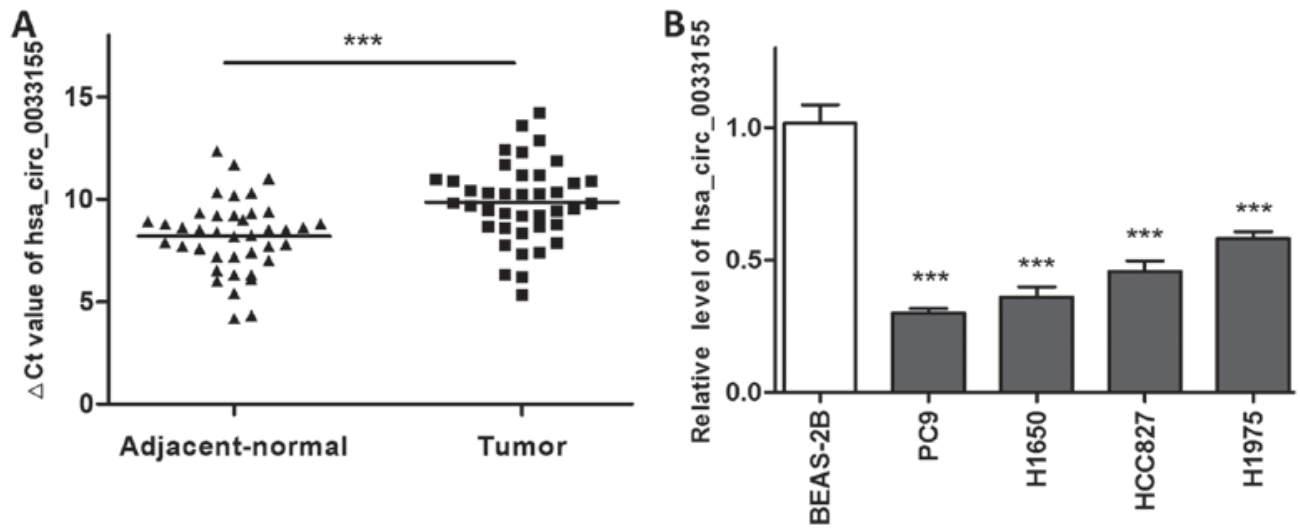

Figure 1. Hsa_circ_0033155 was downregulated in NSCLC tissues and cell lines. (A) Relative $\triangle$ Cq value of hsa_circ_0033155 in 40 pairs of NSCLC tissues and the non-tumorous tissues. ${ }^{* * *} \mathrm{P}<0.001$. (B) The level of hsa_circ_0033155 in NSCLC cells. ${ }^{* * * *} \mathrm{P}<0.001$ vs. BEAS-2B. NSCLC, non-small cell lung cancer.

corresponding monoclonal antibody at $4^{\circ} \mathrm{C}$ overnight followed by incubation with a secondary antibody at RT for $1 \mathrm{~h}$. GAPDH was used as the loading control. Primary antibodies against PTEN (\#9188; 1:1,000), GAPDH (\#5174; 1:1,000) and horseradish peroxidase-conjugated secondary antibody (\#7074; 1:2,000) were obtained from Cell Signaling Technology, Inc. (Danvers, MA, USA). Bands were visualized with an enhanced chemiluminescence kit from Pierce Biotechnology, Inc. (Thermo Fisher Scientific, Inc.).

Statistical analysis. SPSS 16.0 (IBM Corp., Chicago, IL, USA) and GraphPad Prism 5.0 (GraphPad Software, La Jolla, CA, USA) were used for data processing. Data are presented as the mean \pm standard deviation, and each experiment was performed at least three times. Paired and unpaired Student's t-tests were used to analyze significant differences between two groups in tissue samples and cultured cells, respectively. Comparisons of data among multiple groups were performed by one-way analysis of variance followed by Tukey's post hoc test. $\mathrm{P}<0.05$ was considered to indicate a statistically significant difference.

\section{Results}

Hsa_circ_0033155 is downregulated in NSCLC tissues and cell lines. The expression level of hsa_circ_0033155 was initially detected in NSCLC and the matched adjacent non-tumorous tissues using RT-qPCR. As presented in Fig. 1A, the $\triangle \mathrm{Cq}$ values in the NSCLC tissues were significantly higher compared with those in the adjacent non-tumorous tissues, suggesting that the expression of hsa_circ_0033155 in the tumor samples was downregulated. To validate this, the expression level of hsa_circ_0033155 was then measured in four NSCLC cell lines. qPCR results indicated that the levels of circRNA in PC9, H1650, HCC827 and H1975 cells were significantly lower compared with that in BEAS-2B, a normal human bronchus epithelium cell line (Fig. 1B).

Clinical diagnostic value of hsa_circ_0033155 in NSCLC. Subsequently, the association between hsa_circ_0033155 expression levels and clinicopathological characteristics of patients with NSCLC was evaluated. As presented in Table I, the aberrant expression of hsa_circ_0033155 in NSCLC tissues was significantly correlated with lymphatic metastasis
Table I. Associations between hsa_circ_0033155 expression levels and clinical pathological characteristics of patients with NSCLC.

\begin{tabular}{|c|c|c|c|}
\hline Characteristic & $\begin{array}{l}\text { Cases, } \\
\mathrm{n}(\%)\end{array}$ & $\begin{array}{c}\Delta \mathrm{Cq} \\
(\text { mean } \pm \mathrm{SD})\end{array}$ & P-value \\
\hline \multicolumn{4}{|l|}{ Age, years } \\
\hline$\leq 60$ & $24(60.0)$ & $9.86 \pm 1.47$ & \multirow[t]{2}{*}{0.973} \\
\hline$>60$ & $16(40.0)$ & $9.83 \pm 2.56$ & \\
\hline \multicolumn{4}{|l|}{ Gender } \\
\hline Male & $22(55.0)$ & $9.51 \pm 2.22$ & \multirow[t]{2}{*}{0.229} \\
\hline Female & $18(45.0)$ & $10.26 \pm 1.51$ & \\
\hline \multicolumn{4}{|l|}{ Tumor size, cm } \\
\hline$\leq 5$ & $21(52.5)$ & $9.48 \pm 1.72$ & \multirow[t]{2}{*}{0.213} \\
\hline$>5$ & $19(47.5)$ & $10.25 \pm 2.14$ & \\
\hline \multicolumn{4}{|c|}{ Lymphatic metastasis } \\
\hline Yes & $12(30.0)$ & $10.90 \pm 2.27$ & \multirow[t]{2}{*}{0.024} \\
\hline No & $28(70.0)$ & $9.40 \pm 1.63$ & \\
\hline \multicolumn{4}{|l|}{ TNM stage } \\
\hline$\leq \mathrm{II}$ & $18(45.0)$ & $9.83 \pm 1.78$ & \multirow[t]{2}{*}{0.971} \\
\hline$>\mathrm{II}$ & $22(55.0)$ & $9.86 \pm 2.11$ & \\
\hline \multicolumn{4}{|c|}{ Tumor differentiation } \\
\hline Well & $13(32.5)$ & $10.01 \pm 1.85$ & \multirow[t]{3}{*}{0.722} \\
\hline Moderate & $16(40.0)$ & $9.91 \pm 1.95$ & \\
\hline Poor & $11(27.5)$ & $9.57 \pm 2.20$ & \\
\hline
\end{tabular}

$\Delta \mathrm{Cq}=\mathrm{Cq}\left(\mathrm{hsa} \_\right.$circ_0033155) $-\mathrm{Cq}(\mathrm{GAPDH})$. NSCLC, non-small cell lung cancer; SD, standard deviation; TNM, Tumor Node Metastasis.

$(\mathrm{P}=0.0237)$, whereas no significant association was detected between hsa_circ_0033155 expression and other clinicopathological characteristics.

Overexpression of hsa_circ_0033155 decreases cell proliferation. The aforementioned results suggested that downregulated hsa_circ_0033155 may serve a role in NSCLC progression. Therefore, the effects of hsa_circ_0033155 on tumor cell phenotypes were investigated. As relatively lower 


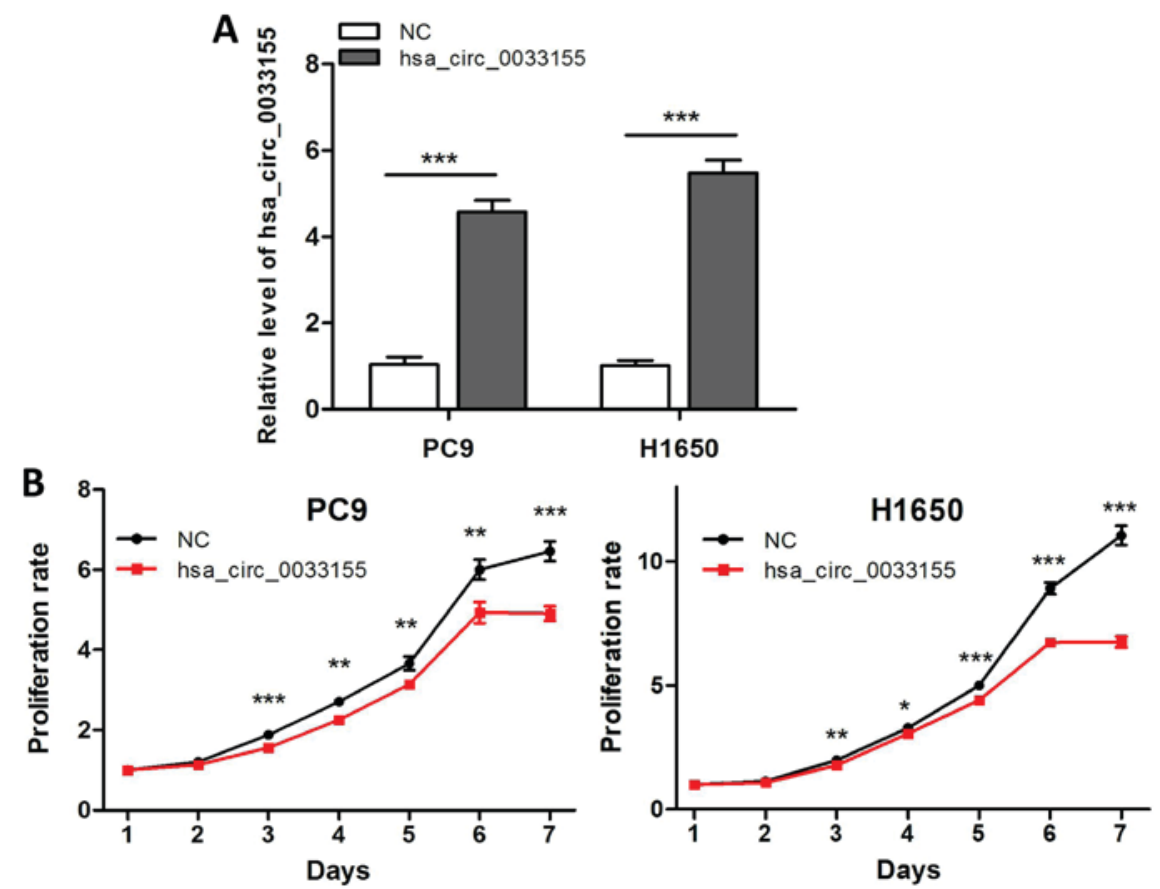

Figure 2. Overexpression of hsa_circ_0033155 decreased cell proliferation. (A) The level of hsa_circ_0033155 in hsa_circ_0033155 overexpressed cells. ${ }^{* * *} \mathrm{P}<0.001$. (B) The effect of hsa_circ_0033155 on cell proliferation. Cells were cultured for 7 days and a MTT assay was applied to detect cell viability daily. ${ }^{*} \mathrm{P}<0.05,{ }^{* *} \mathrm{P}<0.01,{ }^{* * *} \mathrm{P}<0.001$ vs. hsa_circ_0033155. NC, negative control.

A NC hsa_circ_0033155 PC9
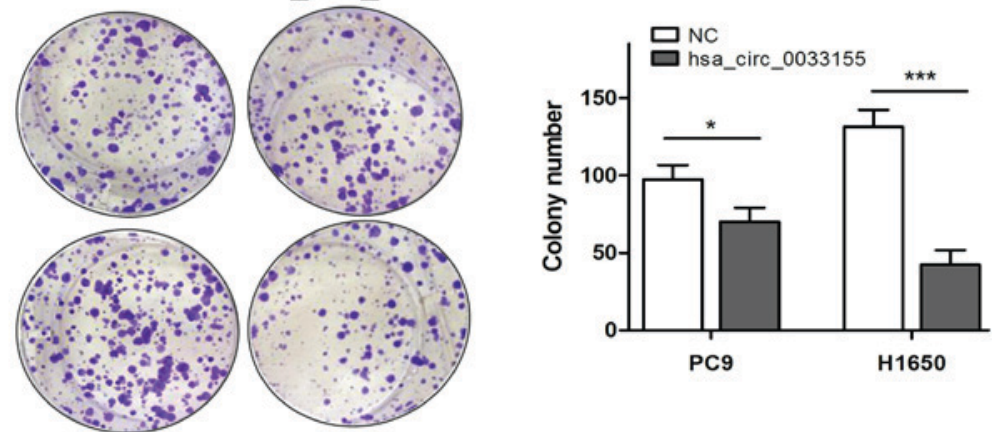

B NC hsa_circ_0033155
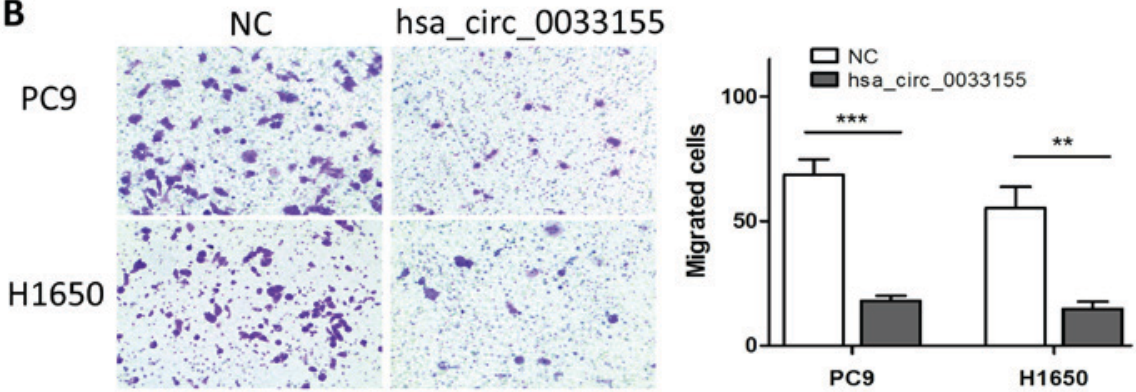

Figure 3. Overexpression of hsa_circ_0033155 inhibited colony formation and migration. (A) Colony formation ability of hsa_circ_0033155-overexpressed NSCLC cells. Cells were cultured for 14 days, then stained, numbered. (B) Migration ability of hsa_circ_0033155-overexpressed NSCLC cells. Cells were cultured for $24 \mathrm{~h}$ in a Transwell chamber, then stained and counted (magnification, $\mathrm{x} 200$ ). ${ }^{*} \mathrm{P}<0.05,{ }^{* *} \mathrm{P}<0.01,{ }^{* * * *} \mathrm{P}<0.001$. NC, negative control.

levels of hsa_circ_0033155 were observed in PC9 and H1650 cells compared with in HCC827 and H1975 cells (Fig. 1B), the former two cell lines were selected to overexpress hsa_circ_0033155. The results of the qPCR assay in Fig. 2A suggested that hsa_circ_0033155 was successfully transfected into the two cell lines. The MTT assay indicated that the overexpression of hsa_circ_0033155 exhibited a significant decrease in cell proliferation following incubation for $>2$ days (Fig. 2B).

Overexpression of hsa_circ_0033155 inhibits colony formation and migration. The effects of hsa_circ_0033155 on colony formation were then determined following incubation for 2 weeks. As presented in Fig. 3A, the overexpression of 

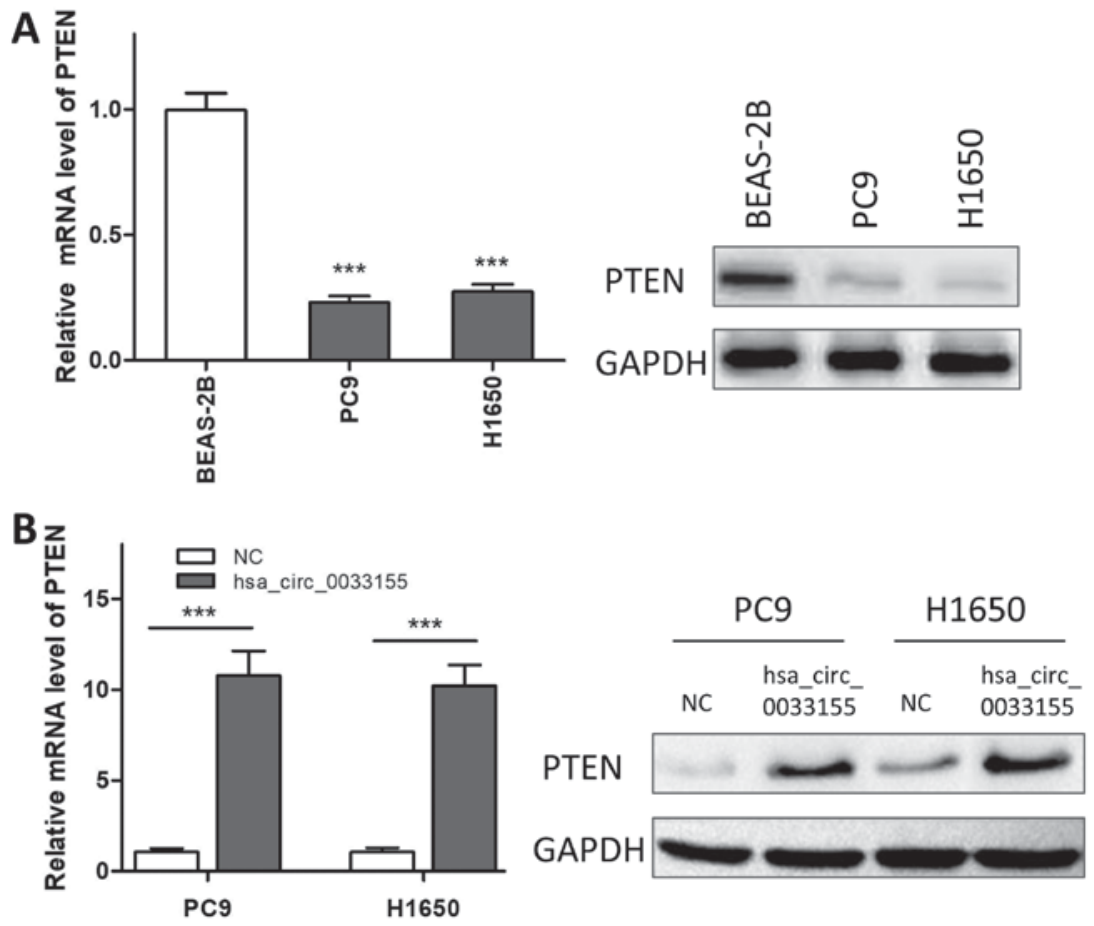

Figure 4. Overexpression of hsa_circ_0033155 upregulated the expression level of PTEN. (A) The mRNA and protein levels of PTEN in BEAS-2B and NSCLC cells. (B) The mRNA and protein level of PTEN in hsa_circ_0033155-overexpressed NSCLC cells. ${ }^{* * *}$ P $<0.001$. PTEN, phosphatase and tensin homolog deleted on chromosome 10; $\mathrm{NC}$, negative control.

hsa_circ_0033155 significantly inhibited the colony formation ability of the two cell lines. The effect of hsa_circ_0033155 on H1650 cells was greater than that on PC9 cells. The effects of hsa_circ_0033155 on the migration ability of cells were also assessed. The number of cells that migrated was significantly inhibited following overexpression of hsa_circ_0033155 (Fig. 3B).

Overexpression of hsa_circ_0033155 upregulates the level of PTEN. As presented in Fig. 4, the mRNA expression of PTEN in PC9 and H1650 cells was significantly decreased compared with that in BEAS-2B cells, which was consistent with the western blotting results (Fig. 4A). The expression level of PTEN was then analyzed in hsa_circ_0033155-overexpressed cells. The results revealed that overexpression of hsa_circ_0033155 significantly increased the expression of PTEN at the mRNA and protein levels (Fig. 4B).

\section{Discussion}

circRNAs are a type of novel untranslated RNA molecule that lacks 5'-3' UTRs and a poly A tail, covalently forming closed loops, which were previously considered to be functionless products of errors in splicing (7). Notably, it is the unique circular structure that protects them from the degradation of exonuclease RNase R and confers these RNAs excellent stability (15). In addition, the expression level of circRNAs, including circular ANRIL, is higher compared with that of linear RNAs $(16,17)$. In addition, their expression level and function are independent of linear RNA isomers $(16,17)$. The aforementioned advantages indicate that, circRNAs may serve as ideal diagnostic biomarkers for cancer, and be superior to other non-coding RNAs such as long non-coding RNAs and miRNAs $(18,19)$.

In the present study, the expression of hsa_circ_0033155 was detected in 40 pairs of NSCLC tissues and the adjacent non-tumorous tissues with qPCR for the first time, to the best of our knowledge. Furthermore, the level of hsa_circ_0033155 was validated in NSCLC cell lines. The results demonstrated that the levels of hsa_circ_0033155 were significantly downregulated in both NSCLC tissues and NSCLC cell lines compared with their corresponding controls. The dysregulated expression was significantly associated with lymphatic metastasis, suggesting the potential value of hsa_circ_0033155 as a biomarker for NSCLC diagnosis. Coincidentally, circRNA_100876 was found to be associated with lymphatic metastasis in NSCLC (20). Therefore, circRNAs including hsa circ_0033155 from NSCLC biopsy samples may be combined with previously reported circ-ITCH and circRNA_100876, which were correlated with tumor staging, to increase positive diagnosis rate (21).

An increasing number of studies have revealed that the aberrant expression of circRNAs is associated with tumor progression. circRNA_100290 is markedly upregulated in oral squamous cell carcinoma tissue, and the knockdown of circRNA_100290 significantly inhibits the proliferative ability of cells (22). In lung cancer, interference of upregulated hsa_circ_0000064 markedly blocked cell cycle progression, promoted cell apoptosis, and decreased migration and invasive activities (23). Therefore, hsa_circ_0033155 was overexpressed in NSCLC cells with lower levels of the circRNA to detect whether the biological functions were attenuated. The results indicated that overexpression of hsa_circ_0033155 significantly decreased cell proliferation and colony formation. Furthermore, 
the migration ability of cells, an important determinant of malignancy progression and metastasis, was also significantly inhibited. These results indicated that hsa_circ_0033155 may serve a cancer-suppressive role in NSCLC progression.

PTEN, a modulator of cell survival and cell cycle progression, has been identified as a tumor suppressor that is downregulated and mutated in various cancers, including hepatocarcinoma, glioblastoma, ovarian and prostate cancer $(13,24,25)$. Therefore, aberrant hsa_circ_0033155 may be associated with the regulation of PTEN, thus regulating cell biological functions. It was also demonstrated that the level of PTEN in NSCLC cell lines was markedly decreased, and that the overexpression of hsa_circ_0033155 significantly enhanced the level of PTEN, revealing the regulatory role of hsa_circ_0033155 on PTEN expression. Emerging studies have demonstrated that circRNA functions as miRNA sponges, removing the inhibitory effect of miRNA on its target genes, and further regulating the expression of target genes, including PTEN $(26,27)$. The miRNA that is regulated by hsa_circ_0033155, subsequently regulating the level of PTEN remains to be investigated.

In conclusion, downregulated hsa_circ_0033155 is associated with lymphatic metastasis in NSCLC and overexpression of hsa_circ_0033155 significantly decreased the proliferation, colony formation and migration abilities of NSCLC cells. Additionally, PTEN may serve an important role in these regulations. Overall, hsa_circ_0033155 may serve as a prospective biomarker and a promising target for NSCLC.

\section{Acknowledgements}

Not applicable.

\section{Funding}

The present study was financially supported by National Natural Science Foundation of China (grant no. 81673014).

\section{Availability of data and materials}

The datasets used and/or analyzed during the current study are available from the corresponding author on reasonable request for non-commercial purposes, without breaching participant confidentiality.

\section{Authors' contributions}

GW and XF worked on the conception and design of the study, and analyzed and interpreted the data. XG and HS collected and assembled data. All authors contributed to preparation of the manuscript and approved the final version.

\section{Ethics approval and consent to participate}

The present study was approved by the Human Research Ethics Committee of Shanghai Jiaotong University (Shanghai, China) and written informed consent was obtained from all patients.

\section{Patient consent for publication}

Not applicable.

\section{Competing interests}

The authors declare that they have no competing interests.

\section{References}

1. Siegel RL, Miller KD and Jemal A: Cancer statistics, 2018. CA Cancer J Clin 68: 7-30, 2018.

2. Tan CS, Gilligan D and Pacey S: Treatment approaches for EGFR-inhibitor-resistant patients with non-small-cell lung cancer. Lancet Oncol 16: e447-e459, 2015.

3. National Cancer Institute: Cancer statistics: SEER fact sheets, lung and bronchus. http://seer.cancer.gov/statfacts/seer.cancer. gov/statfacts/html/lungb.html. Accessed February 5, 2018.

4. Borys Hrinczenko B and Subramonia-Iyer S: Method of biopsy and sample quality for genetic mutation testing in advanced non-small cell lung cancer in a community hospital. J Clin Oncol 30 (34_suppl): S271, 2012.

5. Beermann J, Piccoli MT, Viereck $J$ and Thum T: Non-coding RNAs in development and disease: Background, mechanisms, and therapeutic approaches. Physiol Rev 96: 1297-1325, 2016.

6. Kristensen LS, Hansen TB, Ven $\varnothing$ MT and Kjems J: Circular RNAs in cancer: Opportunities and challenges in the field. Oncogene 37: 555-565, 2018.

7. Han YN, Xia SQ, Zhang YY, Zheng JH and Li W: Circular RNAs: A novel type of biomarker and genetic tools in cancer. Oncotarget 8: 64551-64563, 2017.

8. Han C, Seebacher NA, Hornicek FJ, Kan Q and Duan Z: Regulation of microRNAs function by circular RNAs in human cancer. Oncotarget 8: 64622-64637, 2017.

9. Tian F, Yu CT, Ye WD and Wang Q: Cinnamaldehyde induces cell apoptosis mediated by a novel circular RNA hsa_circ_0043256 in non-small cell lung cancer. Biochem Biophys Res Commun 493: 1260-1266, 2017.

10. Zhao J, Li L, Wang Q, Han H, Zhan Q and Xu M: circRNA expression profile in early-stage lung adenocarcinoma patient. Cell Physiol Biochem 44: 2138-2146, 2017.

11. Chen S, Li T, Zhao Q, Xiao B and Guo J: Using circular RNA hsa_circ_0000190 as a new biomarker in the diagnosis of gastric cancer. Clin Chim Acta 466: 167-171, 2017.

12. Chen Z, Fillmore CM, Hammerman PS, Kim CF and Wong KK: Non-small-cell lung cancers: A heterogeneous set of diseases. Nat Rev Cancer 14: 535-546, 2014.

13. Song MS, Salmena L and Pandolfi PP: The functions and regulation of the PTEN tumour suppressor. Nat Rev Mol Cell Biol 13: 283-296, 2012.

14. Livak KJ and Schmittgen TD: Analysis of relative gene expression data using real-time quantitative PCR and the 2(-Delta Delta C(T)) method. Methods 25: 402-408, 2001.

15. Chen LL: The biogenesis and emerging roles of circular RNAs. Nat Rev Mol Cell Biol 17: 205-211, 2016.

16. Feng J, Xiang Y, Xia S, Liu H, Wang J, Ozguc FM, Lei L, Kong R, Diao L, He C and Han L: CircView: A visualization and exploration tool for circular RNAs. Brief Bioinform: Jun 30, 2017 (Epub ahead of print). doi: 10.1093/bib/bbx070.

17. Qu S, Liu Z, Yang X, Zhou J, Yu H, Zhang R and Li H: The emerging functions and roles of circular RNAs in cancer. Cancer Lett 414: 301-309, 2018.

18. Zhao ZJ and Shen J: Circular RNA participates in the carcinogenesis and the malignant behavior of cancer. RNA Biol 14: 514-521, 2017.

19. Li P, Chen S, Chen H, Mo X, Li T, Shao Y, Xiao B and Guo J: Using circular RNA as a novel type of biomarker in the screening of gastric cancer. Clin Chim Acta 444: 132-136, 2015.

20. Yao JT, Zhao SH, Liu QP, Lv MQ, Zhou DX, Liao ZJ and Nan KJ: Over-expression of circRNA_100876 in non-small cell lung cancer and its prognostic value. Pathol Res Pract 213: 453-456, 2017.

21. Meng S, Zhou H, Feng Z, Xu Z, Tang Y, Li P and Wu M: circRNA: Functions and properties of a novel potential biomarker for cancer. Mol Cancer 16: 94, 2017.

22. Chen L, Zhang S, Wu J, Cui J, Zhong L, Zeng L and Ge S: circRNA_100290 plays a role in oral cancer by functioning as a sponge of the miR-29 family. Oncogene 36: 4551-4561, 2017.

23. Luo YH, Zhu XZ, Huang KW, Zhang Q, Fan YX, Yan PW and Wen J: Emerging roles of circular RNA hsa_circ_0000064 in the proliferation and metastasis of lung cancer. Biomed Pharmacother 96: 892-898, 2017. 
24. Kim C, Lee CK, Chon HJ, Kim JH, Park HS, Heo SJ, Kim HJ, Kim TS, Kwon WS, Chung HC and Rha SY: PTEN loss and level of HER2 amplification is associated with trastuzumab resistance and prognosis in HER2-positive gastric cancer. Oncotarget 8: 113494-113501, 2017.

25. Zhang J, Xu E, Ren C, Yang HJ, Zhang Y, Sun W, Kong X, Zhang W, Chen M, Huang E and Chen X: Genetic ablation of Rbm38 promotes lymphomagenesis in the context of mutant p53 by downregulating PTEN. Cancer Res 78: 1511-1521, 2018.
26. Yang C, Yuan W, Yang X, Li P, Wang J, Han J, Tao J, Li P, Yang H, Lv Q and Zhang W: Circular RNA circ-ITCH inhibits bladder cancer progression by sponging miR-17/miR-224 and regulating p21, PTEN expression. Mol Cancer 17: 19, 2018.

27. Hsu YL, Hung JY, Chang WA, Jian SF, Lin YS, Pan YC, Wu CY and Kuo PL: Hypoxic lung-cancer-derived extracellular vesicle MicroRNA-103a increases the oncogenic effects of macrophages by targeting PTEN. Mol Ther 26: 568-581, 2018. 